\title{
Impact of the COVID-19 Pandemic on Admissions to the Pediatric Emergency Department in a Tertiary Care Hospital
}

\author{
Radhika Raman ${ }^{1} \cdot$ Manoj Madhusudan ${ }^{1}$ (D) \\ Received: 5 September 2020 / Accepted: 12 October 2020 / Published online: 4 November 2020 \\ (C) Dr. K C Chaudhuri Foundation 2020
}

To the Editor: The management of the coronavirus disease (COVID-19) pandemic has centered around the implementation of lockdown and advisories to the general public to stay at home. Though this was an effective strategy to slow the spread of the virus, the anxiety among parents, and the restrictions on travel imposed have influenced the demographic characteristics of hospital admissions. We hence attempted to compare children based on symptomatology at presentation to the pediatric emergency department during the pandemic phase (April-July 2020) with the same period in 2019.

We observed a $65 \%$ drop in admission to the pediatric emergency department in $2020(n=280)$ as compared with a similar time frame last year $(n=790)$. The most significant decrease was observed in admissions due to respiratory $[n=$ $109(13.7 \%)$ to $n=24(8.5 \%) p$ value- 0.023$]$ and gastrointestinal $[n=153(19.3 \%)$ to $n=31(11.1 \%) p$ value- 0.002$]$ emergencies. We also observed that a significantly higher proportion of children admitted into the emergency department required ICU care $[n=56(20 \%)$ in 2020 to $n=76(9.6 \%)$ in $2019 p$ value $<0.0001]$.

With the first case of COVID-19 reported in India on January 30 , there was a growing fear that many children might get sick with the disease in the following days. But rather ironically in a pandemic, we noted a $65 \%$ drop in admissions to our pediatric emergency department. A similar trend has been observed in multiple centers world over [1,2]. The sharp decrease in respiratory and gastrointestinal emergencies could be attributed to various reasons such as a reduction in vehicular pollution, improved hand hygiene, and universal masking. Another additional factor could be the closure of schools and other public places, reducing the spread of infections among children. A significant increase in intensive care requirements was observed among children admitted during the pandemic. In a similar observation from Italy, Lazzarini et al. [3] in their study of 12 children with delayed access to health care noted that in all cases, parents avoided accessing healthcare because of the fear of getting COVID infection.

The life style changes and restrictions associated with the COVID-19 pandemic had resulted in a reduction in admissions to the pediatric emergency department but an increase in delayed presentation and ICU requirement.

\section{Compliance with Ethical Standards}

Conflict of Interest None.

\section{References}

1. Scaramuzza A, Tagliaferri F, Bonetti L, et al. Changing admission patterns in pediatric emergency departments during the COVID-19 pandemic. Arch Dis Child. 2020;105:704-6. https://doi.org/10.1136/ archdischild-2020-319397.

2. Roland D, Harwood R, Bishop N, Hargreaves D, Patel S, Sinha I. Children's emergency presentations during the COVID-19 pandemic. Lancet Child Adolesc Health. 2020;4:e32-3. https://doi.org/10. 1016/S2352-4642(20)30206-6.

3. Lazzerini M, Barbi E, Apicella A, Marchetti F, Cardinale F, Trobia $\mathrm{G}$. Delayed access or provision of care in Italy resulting from fear of COVID-19. Lancet Child Adolesc Health. 2020;4:e10-11.

Publisher's Note Springer Nature remains neutral with regard to jurisdictional claims in published maps and institutional affiliations.

Manoj Madhusudan

manoj-93@live.in

1 Department of Pediatric Emergency Medicine, Kanchi Kamakotti CHILDS Trust Hospital, Chennai, Tamil Nadu, India 